\title{
Une méthode théologique aux prises avec le statut herméneutique de la foi
}

\author{
A theological method dealing with the \\ hermeneutical status of faith
}

\section{Pedro Rubens*}

Universidade Católica de Pernambuco (UNICAP), Recife, Pernambuco, Brasil

\section{Résumé}

Pour accomplir la triple tâche de la théologie fondamentale - interprétative (médiation herméneutique), réflexive (réflexion épistémologique) et auto-implicative (apologie de la différence chrétienne), l'auteur propose une méthode en tant que discernement de la foi chrétienne au sein des expériences religieuses ambiguës. L'article explicite d'abord le point de départ de la réflexion et son importance pour la méthode théologique ; ensuite il engage un dialogue avec deux interlocuteurs - Paul Tillich et Clodovis Boff - pour indiquer les démarches d'une théologie aux prises avec les situations contextuelles; et puis il trace quelques pistes d'une méthode herméneutique et contextuelle.

Mots-clés: Méthode.Théologie Fondamentale. Discernement. Contexte. Herméneutique.

* PR: Doutor em Teologia, e-mail: pedro_rubens@hotmail.com 


\section{Abstract}

In order to carry out the triple task of fundamental theology: interpretative (hermeneutical mediation), reflexive (epistemological reflection) and self-implicative (apology of the Christian difference), the author proposes a method for the discernment of the Christian faith in the context of ambiguous religious experiences. The article first explores the starting point of the reflection and its relevance for the theological method; it then establishes a dialogue with two interlocutors - Paul Tillich and Clodovis Boff - to point out the approaches to a theology grappling with contextual realities; it outlines some considerations for a hermeneutical and contextual method.

Keywords: Method. Fundamental Theology. Discernment. Context. Hermeneutics.

\section{Introduction ${ }^{1}$}

Soyez toujours prêts à justifier votre espérance devant ceux qui vous en demandent compte $(1 \mathrm{P} 3,15)$ : cette attitude concerne le chrétien et la théologie en général, mais elle s'adresse plus spécifiquement à la théologie fondamentale, héritière d'une tradition aussi ancienne que le propre christianisme. La théologie fondamentale (SESBOÜE; THEOBALD, 1996; SILVA, 1998, p. 111-149) cherche à définir ses tâches à partir d'une double fonction qui caractérise sa propre méthode: elle doit traiter des présupposés du christianisme (praeambula fidei), en se servant non seulement d'études philosophiques et historiques l'aidant à établir sa crédibilité devant le tribunal de la raison humaine, mais aussi des religions du monde et des différentes confessions chrétiennes (premier pôle) ; en même temps, elle doit se comprendre comme une discipline éminemment « théologique » fondée sur la possibilité et la nécessité d'une démonstration rationnelle de la foi sur sa propre Révélation chrétienne (second pôle) (THEOBALD, 1996, p. 228).

\footnotetext{
1 Le présent texte revisité correspond à l'exposé fait par moi-même aux doctorants en théologie de l'Institut Catholique de Paris dans le cadre d'une session de rentrée : c'est la raison d'avoir gardé la réflexion dans la langue de la communication réalisée.
} 
Notre perspective est ici méthodologique et consistera en une réinterprétation contextuelle de cette double fonction de la théologie fondamentale ${ }^{2}$, selon sa triple tâche : interprétative (médiation herméneutique), proprement réflexive (réflexion épistémologique) et auto-implicative (apologie de la différence chrétienne), dans un exercice de discernement théologique et pastoral. Autrement dit, il s'agira dans un premier temps d'interpréter les expériences d'un contexte donné, afin de réfléchir par la suite aux médiations et critères interprétatifs qui répondent tout autant à la réalité qu'à l'exigence d'une théologie chrétienne ; enfin, dans un troisième temps, il faudra élaborer une apologie de la foi, en dialogue avec d'autres formes de pensées et d'autres styles de vie.

Concrètement, je me propose de mettre en évidence quelques pistes méthodologiques, moins pour élaborer une théorie de la méthode ${ }^{3}$ que pour relire le chemin parcouru dans le cadre d'un travail de thèse ${ }^{4}$ organisé en trois parties. La première, notre point de départ, consiste en une interprétation des expériences contextuelles moyennant une description phénoménologique et une analyse socioreligieuse, sans omettre pour autant les questions que la médiation socio-analytique suscite : en plus du passage des analyses sociologiques au discours théologique, nous avons aussi soulevé l'hypothèse du propre statut " herméneutique » et contextuel de l'acte de croire (RUBENS, 2004, p. 23-134). La deuxième partie implique, quant à elle, une prise de distance par rapport à la situation contextuelle pour " penser avec » (RUBENS, 2004, p. 135-322) Paul Tillich une "critériologie » en vue d'un discernement théologique. Cependant, il faut avouer que ma relecture de Tillich ne se fait pas sans une précompréhension donnée par l'horizon des théologies contextuelles

2 Aussi bien dans le contexte large de la théologie contemporaine que dans le contexte spécifique d'une théologie " régionale ". Voir l'œuvre de Waldenfels (1990); Kern et al. (1990).

3 En théologie contemporaine, la référence est l'ouvrage de Lonergan (1972). Dans le contexte latino-américain il y a aussi eu de nombreuses recherches concernant la méthode de théologie, parmi lesquelles : Boff (1990 ; 1998/2012); Taborda (1987, p. 293-319); Scannone (1994, p. 255-283).

4 Une méthode, c'est avant tout un chemin ; et un chemin se fait en cheminant. II en va de même en théologie: un discours sur la méthode suppose qu'un parcours ait déjà été fait. En tout cas, ce travail est une réflexion sur le chemin tracé lors de l'élaboration de la thèse de doctorat en théologie systématique que j'ai soutenue aux Facultés Jésuites de Paris et qui a été publiée en janvier 2004 sous le titre Discerner la foi dans des contextes religieux ambigus: enjeux d'une théologie du croire (RUBENS, 2004). 
latino-américaines : c'est pourquoi un débat avec Clodovis Boff et avec la méthode des théologies de la libération devient important. Enfin, la troisième partie correspond à une auto-compréhension du christianisme, postulée comme une " apologie de la différence chrétienne ». Aussi ancienne que la foi elle-même, la fonction apologétique de la théologie indique en effet un critère de notre rapport à l'autre. Si une longue histoire de définition de l'identité chrétienne s'oppose à l'autre, comme le montre à la fois les apologies et l'apologétique moderne, notre rapport à l'autre doit aujourd'hui s'interpréter à la lumière de l'ouverture au différent, du dialogue avec autrui et du pluralisme des conceptions. Le plus grand défi d'une apologie de la différence chrétienne est donc de reconnaître l'autre en tant qu'autre, dans son irréductible différence, sans renoncer pour autant à son identité chrétienne, dans sa singularité radicale (RUBENS, 2004, p. 323 et seq.).

\section{Point de départ : l'expérience de la foi à une époque d'ambiguïté religieuse}

La question du point de départ est importante parce qu'il ne s'agit pas d'un simple commencement, mais d'un lieu herméneutique à partir duquel s'ouvrent, non seulement la perspective de lecture théologique de la réalité à la lumière de la Parole de Dieu, mais [...] aussi le point de vue à partir duquel cette même Parole et les contenus de la foi de l'église seront interprétés (SCANNONE, 1994, p. 260).

À la lumière de cette considération judicieuse de J. C. Scannone, nous reconnaissons deux héritages dans la définition de notre point de départ : d'un côté, les études des théologies européennes sur l'expérien$\mathrm{ce}^{5}$, exigence du tournant anthropocentrique de la modernité occidentale et expression de la direction prise par le Concile Vatican II ; de l'autre, la piste de recherche méthodologique des théologies latino-américaines de la libération (LIBANIO, 1989) ${ }^{6}$, également en dialogue avec la tradition européenne, mais insérée dans une perspective de réception créative et

5 La bibliographie sur l'expérience en théologie est assez vaste, surtout depuis le Concile Vatican II. Cf. Bouillard (1965, p. 83-92) ; Schillebeeckx (1981, p. 29-64 ; 1992, p. 23-87) ; Moltmann (1999, p. 37-113).

6 L'auteur y présente une diversité des méthodes et une pluralité des styles à l'intérieur même de la théologie latino-américaine. Voir également Ellacuria et Sobrino (1990). 
d'ouverture de chemins non tracés. Nous sommes donc partis d'une espèce de description phénoménologique de l'expérience contextuelle du croire, là où et comment la foi se manifeste de la manière la plus visible. Bien sûr, ce point de départ n'épuise pas toute la richesse de la foi qui, d'une certaine manière, n'est accessible qu'à Dieu qui rencontre les êtres dans le secret (GISEL, 1989, p. 63 et seq.).

\section{La dimension religieuse et culturelle de la foi}

Le catholicisme latino-américain vit une situation extrêmement ambiguë : d'une part, il présente des signes de déclin propres à la civilisation chrétienne occidentale et à l'éclipse de la religion comme référentiel de la société ; d'autre part, la foi chrétienne est affectée par le resurgissement du religieux sous ses formes les plus archaïques, au sein même de la vie contemporaine. Pour certains, cette poussée religieuse est typique d'une époque d'incertitudes et de peurs. En effet, les formes de violence et les motifs alimentant le climat d'insécurité se multiplient chaque jour, autant dans la sphère collective que dans la sphère individuelle, à l'échelle planétaire aussi bien que locale. Pour d'autres, ce phénomène religieux n'est qu'un « retour de la religion », sorte de « revanche du sacré » qui, bien que correspondant à une dimension constitutive de l'être humain, fut critiqué et réprimé par la raison moderne qui prophétisa le dépassement du stade religieux de l'humanité. Le débat est toujours en cours, mais nous pouvons déjà entrevoir l'obstacle de nos préjugés et une certaine inadéquation de nos vieilles catégories " modernes ", jugées par les post-modernes comme totalisantes, voire même exclusivistes ${ }^{7}$.

Il semble toutefois évident qu'il y ait une véritable montée du religieux et un intérêt renouvelé pour le " spirituel $^{8}$ ", qui vient à l'encontre de la plupart des prévisions des prophètes de la modernité occidentale. Néanmoins, cela ne contredit pas les diagnostics de " fin de la religion ",

Sur la théologie et la postmodernité voir l'ouvrage magistral du théologien mexicain Carlos Mendoza-Alvarez (2011), 0 Deus escondido da pós-modernidade. Cet essai de théologie fondamental post-moderne a également été traduit par les Éditions du Cerf sous le titre Deus absconditus.

8 Les termes spiritualité, religiosité, voire même mystique sont employés indistinctement. Le vocabulaire luimême mériterait un discernement ou, pour le moins, une plus grande précision. 
toutefois une plus grande précision est de rigueur : le problème n'est pas celui de la disparition des religions, mais bien celui de la fin d'une époque où elles représentaient, d'une forme ou d'une autre, le fondement de la vision du monde ou la norme de l'organisation sociale. Cette fonction ne lui étant plus attribuée, nous devons nous interroger sur cette " fin », dans le sens d'une téléologie de la dimension religieuse de l'être humain et de la religion, dans l'histoire de l'humanité. Cependant, cette montée religieuse actuelle n'autorise aucune religion à rêver à un retour des théocraties historiques ni ne permet au christianisme occidental de revendiquer un statut politique privilégié dans les sociétés contemporaines dites «post-chrétiennes ». Par ailleurs, l'épuisement du rôle joué par le christianisme en Occident ne signifie pas que la théologie doive se sentir "déresponsabilisée » face aux grands débats de la vie moderne, aux problèmes plus personnels et aux défis d'une société qui se mondialise.

Ces questions vitales concernent le christianisme dans la mesure où elles impliquent l'être humain dans sa dignité inaliénable. Indépendamment du statut social de la religion, ceci est une conséquence de la propre dynamique de l'Incarnation, sans laquelle le christianisme ne serait pas lui-même : comme le disaient les Pères de l'Eglise, ce qui n'a pas été assumé par le Verbe qui s'est fait chair n'a pas été sauvé. La tâche de la théologie est de rendre raison de notre espérance (cf. 1Pd 3,15), mais, avant toute chose, le propre de la foi chrétienne est " d'espérer contre toute espérance » $(\mathrm{Rm} 4,18)$ et de contribuer courageusement et humblement, d'une manière efficace et modeste, à la reconstruction des identités régionales, à l'histoire commune de l'humanité et au destin de la planète.

Cependant, même en assumant la réalité humaine dans sa totalité et en dépassant les frontières du cadre religieux, le christianisme ne peut pas renier son rapport à la religion, surtout dans un contexte où les formes religieuses les plus diverses prétendent répondre aux vrais problèmes par de fausses solutions. En effet, certains discours et pratiques religieuses invoquant le nom de Dieu peuvent servir à légitimer des situations de misère et de souffrance tout autant que des stratégies politiques, voire idéologiques, destructrices. Face à une telle situation, qui n'est pas complètement nouvelle, la notion dialectique du christianisme comme « religion du dépassement de la religion » (TILLICH, 1991), " religion de 
la fin de la religion » (GAUCHET, 1985) ou « religion de l'interprétation » (THEOBALD, 1990, p. 111-132) reste indispensable pour repenser les enjeux et les risques de cette dimension pleine d'ambiguïté de l'être humain. Cela nous conduit à postuler le christianisme comme la religion du discernement de la religion.

\section{Foi chrétienne et ambiguïté religieuse au Brésil}

Dans le contexte brésilien, nous avons identifié et étudié les trois expressions religieuses "chrétiennes " les plus représentatives, qui entrent en scène à l'époque postconciliaire, à savoir : les Communautés ecclésiales de base (Cebs), la Rénovation Charismatique Catholique (RCC) et plus récemment, les néo-pentecôtistes (RUBENS, 2004, p. 23-107)9 . Ces expériences aux visages multiples ont été interprétées aussi bien du point de vue de leur "prétention » de renouveler la foi chrétienne que de celui des ambiguïtés par rapport aux instances de régulation de la foi, plus particulièrement celles concernant l'Eglise catholique. Certes, en tant que figures historiques, elles sont récemment passées par des mutations (LIBANIO, 2002 ; 2003), mais elles demeurent importantes pour la compréhension de la foi en situation.

Après la tentative d'un renouveau de la foi en dialogue avec les réalités sociales et historiques, dans la lignée de l'aggiornamento postconciliaire, l'impact le plus fort pour le christianisme au Brésil, et peut-être même pour toute la région sud-américaine, est incontestablement cette effervescence religieuse ${ }^{10}$, accompagnée d'une ambiguité dépassant les limites exigées par un discernement sérieux, aussi difficile que nécessaire. Nécessaire, car l'exigence chrétienne de recherche de la vérité concernant Dieu et l'humanité est une tâche permanente de la théologie. Difficile, car la réalité est complexe et n'implique pas uniquement une analyse de la situation : il y a un mélange d'éléments modernes et archaïques au point

9 L'étude de ces trois expériences fait l'objet de la première partie de l'ouvrage (RUBENS, 2004). Je ne reviens au thème en question que pour indiquer les éléments du chemin méthodologique parcouru dans ma thèse, sans pouvoir ici réflechir davantage sur les expériences étudiées.

10 Sur le catholicisme populaire, voir : Oliveira (1972, p. 567-584); OLIVEIRA, P. R. et al. Catolicismo popular no Brasil, Revista Eclesiástica Brasileira, v. 36, n. 141, 1976 (Cf. bibliographie, p. 272-280). 
où l'on en vient à confondre le spiritualisme light et le religieux sauvage. En plus des nombreuses expressions prémodernes présentes dans la religiosité populaire et des ambiguïtés de la vie en général, le processus d'évangélisation chrétienne a aussi laissé ses empreintes dans l'histoire du continent. Celles-ci se confondent souvent avec l'importation des processus civilisateurs, non moins ambigus, impliquant des modèles politiques et culturels exogènes.

À titre d'illustration, nous pouvons parler de la relation entre le « catholicisme populaire » et le « catholicisme officiel » qui reflète le problème d'une tension mal résolue et qui, pour cela même, revient sur scène sous les formes les plus inattendues, singulièrement archaïques. En effet, la religiosité populaire joue un rôle de " mémoire vivante " (HERVIEU LEGER, 1987, p. 315et seq ; 1993) des différents moments de l'évangélisation, y compris dans ses formes syncrétiques. Ambigu par excellence, le catholicisme populaire est une forme de religiosité et surtout la matrice de l'expérience socioculturelle et religieuse du Brésil ; matrice dans son sens premier de « sein maternel » ou « d'utérus ». Nous postulons, dans ce contexte brésilien, une relation de "filiation » du catholicisme populaire avec trois figures importantes de l'époque postconciliaire, engendrées par un ensemble de facteurs ecclésiaux, sociaux, économiques et idéologiques.

Tout d'abord, les Cebs, qui, en rupture avec le catholicisme populaire traditionnel, ont cherché à réinventer le populaire en proposant une « nouvelle manière d'être Eglise ». Ensuite, la RCC, mouvement «importé ", initialement associé à la classe moyenne, mais qui a vite acquis des caractéristiques locales et conjugue une libération intérieure et émotionnelle avec des formes d'expression issues du catholicisme traditionnel ; on y observe notamment une grande nécessité d'auto-affirmation par rapport à ses racines pentecôtistes non-catholiques. Enfin, les néo-pentecôtistes ou les églises pentecôtistes de la dernière génération qui se développent selon un modèle de fragmentation et de création constante de nouvelles églises, caractéristiques typiques du mouvement de la Réforme, mais qui, de façon surprenante, incorporent des symboles et des expressions de la religiosité populaire : par exemple, les chaînes de prière, l'onction des malades, les exorcismes, etc. À tout cela, on doit aussi ajouter des éléments de moralité et une vision magique et apocalyptique du monde. 
Malgré leurs différences irréductibles, ces trois figures contextuelles ont des caractéristiques communes. Un point fondamental est qu'elles ont toutes la prétention de "rénover » le christianisme, en faisant appel à un « retour aux sources » et au dynamisme de l'Esprit : les Cebs en rappelant les premières communautés chrétiennes (At 2) ; la RCC en récupérant l'imaginaire de charismes particuliers et en s'identifiant à une " nouvelle Pentecôte "; les néo-pentecôtistes, quant à eux, et à l'intérieur du même univers, en tablant sur le caractère performatif de la parole qui réalise immédiatement et miraculeusement ce qu'elle proclame.

Synthétiquement, nous pouvons identifier quatre grands foyers de tension : premièrement, même si l'on admet que la Sainte Écriture est la " source " commune de ces trois figures, leur rapport au texte et leur mode d'interprétation sont radicalement différents ; deuxièmement, toutes trois diffèrent dans leur relation entre théorie et action, entre le dire et le faire, ou, dans un langage plus élaboré, entre orthodoxie et orthopraxie ; le troisième foyer de tension s'exprime dans le rapport existant entre charisme et institution, tradition et utopie ; enfin, le dernier foyer de tension apparaît dans le rapport entre unité et diversité, unicité de la foi et pluralisme des expressions, et, en dernière instance, dans le rapport entre Jésus-Christ et l'Esprit Saint.

Ces quatre facteurs de tension ainsi que l'ambiguïté de leurs expériences ayant été identifiés, nous devons procéder avec le discernement nécessaire. La plus grande difficulté commence avec la recherche des critères: si, d'un côté, on part de critères purement objectifs (« dogmatiques ») et extérieurs au "monde » des expériences, on court le risque de ne pas rendre compte de leur possible "nouveauté » et de ne pas faire justice au réel ; si, de l'autre, on part de la valorisation de toutes les expériences, aussi diverses soient-elles, travail qui serait exhaustif, on court le risque de tomber dans un relativisme où " tout vaut la peine ", sans aucun critère de vérité possible. Plus qu'un dilemme, ce défi correspond à un paradoxe fondamentalement chrétien : comment rendre compte de l'expérience humaine, dans son irréductible singularité, sans renoncer pour autant à l'affirmation de l'unicité de la foi chrétienne dans son incontournable radicalité ? Dit de manière positive : Dieu s'est révélé pleinement et une fois pour toute, dans la singularité d'un homme situé historiquement, 
Jésus-Christ ; cependant, cette révélation concerne tout être humain, de tous les temps et de toutes les cultures. Ainsi, le discernement quitte la sphère circonstancielle et l'environnement simplement contextuel pour atteindre les fondements de l'acte de croire.

\section{Penser avec Paul Tillich la corrélation entre " situation " et « message "}

La méthode pratiquée par P. Tillich est née d'une interprétation de son propre contexte théologique : le débat et la situation historique de notre auteur ont été, d'un côté, marqués par la sécularisation et, de l'autre, par le « biblicisme » orthodoxe qui réduisait l'importance ou niait explicitement la médiation culturelle dans la construction de la méthode théologique.

En l'appelant " méthode de corrélation », l'auteur établit une interaction dialectique entre "message chrétien » et " situation ». Par cette dernière, entendons non seulement toute et n'importe quelle expression culturelle, fondamentalement philosophique, mais aussi l'expression issue des sciences humaines et des manifestations artistiques ${ }^{11}$. Selon lui, la théologie naît donc de la " corrélation » entre les grandes questions humaines, élaborées par les expressions culturelles et les réponses de la foi, présentes dans les grands "symboles » chrétiens ${ }^{12}$. Il s'agit d'une réponse ouverte, parce qu'un symbole a sans cesse besoin d'être interprété. Audelà de l'ouverture proposée par sa méthode, l'auteur allemand propose aussi, dans un petit essai sur la foi (TILLICH, 1957), une vision « dynamique » de l'expérience croyante qui, comme j'essaye de le montrer, est enracinée dans sa pneumatologie : la dynamique de la foi est donnée par l'Esprit (RUBENS, 1994, p. 285 et seq.). Il y a, de surcroît, une autre raison déterminante qui m’a amené à choisir cet interlocuteur : le dynamisme de la foi est immergé dans l'ambiguïté de l'expérience culturelle et religieuse.

11 Cette ouverture se voit surtout dans la phase américaine de la pensée de Tillich durant laquelle il "systématise" sa méthode de corrélation (RUBENS, 2004, p. 219-231).

12 Le projet de sa théologie systématique suit ce schéma, comme on peut le remarquer par les titres des cinq parties de son système : le premier thème indique toujours l'élaboration culturelle de la question, le second le symbole chrétien qui doit être réinterprété. Ainsi, nous avons : 1. Raison et Révélation ; 2. L'être et Dieu ; 3. L'existence et le Christ ; 4. La vie et l'Ésprit ; 5. L'Histoire et le Règne de Dieu. Cf. Tillich (1991, p. 66-67) ; Rubens (2004, p. 203 et seq.). 
Tillich conduit ainsi l'ambiguïté jusqu'à ses ultimes limites, la déplaçant du cadre religieux à celui de la vie : l'expérience religieuse est ambiguë, parce que la vie elle-même est ambiguë. Tillich radicalise l'ambiguïté, la renvoyant à l'anthropologie théologique ${ }^{13}$. Dans ce sens, l'ambiguïté de l'expérience ne peut pas être éliminée sans courir le risque de tomber dans un idéalisme de l'être humain et de compromettre la propre expérience de foi comme expérience humaine de Dieu. C'est précisément en réinterprétant le symbole chrétien de l'Esprit, Seigneur de la vie, que Tillich entend répondre au problème de l'ambiguïté de la vie.

Dans la démarche méthodologique, Tillich a vu qu'il fallait «traduire » les notions bibliques en catégories de la culture au sens large du terme et c'est de là que provient sa " théologie de la culture ». C'est dans son projet de "théologie systématique " que sa méthode atteint son expression la plus raffinée. Selon Tillich, la construction du discours théologique suppose une notion plurielle de " source ", à savoir : la Bible, l'histoire des Églises et l'histoire religieuse et culturelle. Deux remarques préliminaires s'imposent : tout d'abord, l'auteur suppose une " hiérarchie " parmi ces sources, pour ne pas retomber dans le vieux problème catholique-protestant des "deux sources ", entendons par là les Écritures et la Tradition. Évidemment, les Écritures sont considérées comme « la » source par excellence de la théologie et de la vie chrétienne ; mais l'histoire de l'Église, des religions et des cultures interfèrent aussi de manière décisive dans l'élaboration du discours théologique ${ }^{14}$; par ailleurs, ces "sources » ont effectivement contribué à la propre formation du corpus biblique. Pour Tillich, "source » a donc un sens qui se rapproche de celui de lieu théologique ou encore de «lieu herméneutique ${ }^{15}$.

13 Une comparaison des positions de P.Tillich e de K. Barth me paraît très utile : le débat entre la foi et la religion se heurte à une conception anthropologique différente. Sur ce débat entre les deux auteurs, voir Rubens (2004, p. 152 et seq.).

14 II est important de souligner que, d'une certaine manière, pour Tillich, l'histoire, dans son nouveau statut de science, semble être la grande " médiation " ou " le lieu théologique " par excellence ; l'interprétation des Écritures, dans la vision et à l'époque de Tillich, est marquée par les méthodes historiques critiques ; luimême considérait la Bible comme un "évènement et un document original " (TILLICH, 1991, p. 35).

15 Scannone observa, à juste titre, que l'on emploie ces expressions dans un sens général et qu'il conviendrait de dire non pas "lieu théologique " mais " lieu herméneutique ", et que cette dernière expression a besoin d'un discernement avant d'être employée par la théologie (1994, p. 264). 
Sans vouloir faire un procès critique à la façon de procéder tillichienne, il est important de nous demander jusqu'à quel point sa méthode répond encore aux défis actuels. Bien sûr, quelques aspects demeurent ou ont même été renforcés. Par exemple : les cultures occidentales modernes et post-modernes dépassent l'horizon chrétien; les Saintes Écritures ne sont plus considérées comme le " texte fondateur " du sens de la vie et de la société. Cependant, le contexte est radicalement différent et beaucoup plus complexe. On observe donc, au-delà de la mentalité historiciste et positiviste, une nouvelle ouverture et un élargissement de l'horizon du sens, grâce aux nouvelles études herméneutiques de valorisation du texte comme œuvre et grâce aussi à une mentalité de plus en plus pluraliste. Sous quelque forme que ce soit, une traduction de la Bible dans le langage de la culture qui ne respecterait ni l'altérité du texte biblique, ni l'autonomie des expressions culturelles se révélerait insuffisante.

Cependant, le travail d'inculturation de l'Évangile et l'actualisation de la foi sont bien plus complexes et d'une plus grande radicalité : il ne s'agit pas seulement d'une question de changement des "représentations " ou de la «traduction » de catégories, mais d'une véritable " conversion ", éliminant le caractère d'extériorité des relations entre foi et culture, chacune d'entre elles comprise dans sa pluralité de formes et d'expressions $^{16}$. La justification méthodologique des "sources historiques » dans le discours théologique de Tillich nous mène, d'une certaine manière, vers la perspective développée par les théologies latino-américaines, qui ont donné au « contexte historique » le rang de locus theologicus (RUBENS, 2004, p. 375et seq.).

\section{Penser avec Clodovis Boff le rapport entre théorie et pratique}

Avec rigueur, finesse et sens critique, Clodovis Boff a développé, à des moments différents de sa vie, une réflexion sur les pas méthodologiques

\footnotetext{
${ }^{16}$ Voir l'ouvrage collectif " 50 ans après le Concile Vatican II : Des théologiens du monde délibèrent ". Rome : Libreria Editrice Vaticana/FIUC, 2015, surtout le chapitre 3 : L'unité et la diversité dans la rencontre de l'Évangile et de l'Église avec le monde et les cultures (LAMBERIGTS et al., 2015, p. 85-104).
} 
faits par les théologies de la libération ${ }^{17}$. Suivant la piste de la méthode pastorale voir-juger-agir, employée par l'Action Catholique (BRIGHENTI, 1994, p. 207-254), C. Boff essaye d'articuler - déjà dans un niveau théorique - les trois moments nécessaires à l'élaboration de ce qu'il appelle une " théologie du politique " (BOFF, $1978 ; 1990)^{18}$. Postérieurement, l'auteur précisera sa conception méthodologique (BOFF, 1998/2012 ; $\mathrm{ADÃO}, 2015)^{19}$ de manière plus approfondie et didactique.

Voyons, en résumé, les étapes de la constitution d'une théologie du politique. Dans un premier temps, l'auteur justifie la nécessité d'une médiation socio-analytique pour interpréter la réalité contextuelle de la pauvreté en Amérique Latine - et ceci afin de développer une action chrétienne conséquente : l'objet à appréhender indique donc à la fois la méthode et la médiation. Dans un deuxième temps, $C$. Boff appelle « médiation herméneutique » la confrontation avec les Écritures chrétiennes : «Il s'agit d'un moment théologique nécessaire (pas le seul) pour la construction intégrale d'une théologie (chrétienne) (BOFF, 1990, p. 120)». Dans cette démarche d'interprétation et d'actualisation des Écritures, l'auteur analyse d'une manière critique quelques modèles d'interprétation des Écritures dans les théologies de la libération avant de proposer son modèle herméneutique.

Boff met en question le modèle des "termes correspondants ", selon le schéma simplifié sous la forme d'une équation à résoudre comme suit (BOFF, 1990, p. 242-249; 244-245) :

${ }_{17}$ On observe la conception plurielle des théologies de la libération: voir (LIBANIO, 1987). L'(auto)critique de Clodovis Boff aux "fondements " méthodologiques des théologies de la libération (en 2007) ne change pas l'orientation fondamentale de sa réflexion précédente (ce que nous exposerons maintenant). Sensible à certaines dérives locales du tournant anthropocentrique en théologie, Clodovis exigera de ses collègues une affirmation toujours plus nette de la place de la foi en Jésus-Christ comme principe méthodologique de toute théologie. Ainsi, le rapport entre théorie et pratique ne doit pas être considéré comme une question simplement méthodologique, car le théologien doit avoir dans l'esprit, avant tout, une praxis originaire : l'action de Dieu pour le salut du monde. À ce sujet, voir Francys Adão. Nossa parte da herança: os frutos de um debate teológico no Brasil. REB. Revista Eclesiástica Brasileira, v. 74, p. 264-299, 2014.

18 Dans l'expression " théologie du politique " (au lieu de " théologie politique "), nous remarquons la distinction et l'effort d'articulation entre théorie (théologique) et pratique (politique) qui traversera toute sa réflexion.

19 Voir Francys Adão. Por uma hermenêutica bíblica pneumática: Clodovis Boff e a hermenêutica da libertação. Rio de Janeiro, Atualidade Teológica, v. 49, p. 143-156, jan./abr. 2015. 


\begin{tabular}{|r|l|l|}
\hline Écriture & $=$ & Théologie du Politique \\
\hline Exode situation politique & $=$ & Notre situation politique \\
\hline Esclavage des Hébreux & $=$ & Théologie de la Libération \\
\hline Babylone & $=$ & Oppression du Peuple \\
\hline Israël & $=$ & Peuple d ' Amérique latine \\
\hline Jésus & $=$ & Communauté chrétienne \\
\hline Son contexte politique & $=$ & Contexte politique actuel \\
\hline
\end{tabular}

Selon Boff, ce modèle risque d'établir une correspondance immédiate, de ne pas prendre en compte les singularités des contextes bibliques et moins encore la complexité de nouveaux contextes (BOFF, 1990, p. 248-249). Il propose donc un modèle alternatif et à la fois correctif du modèle des "termes correspondants " : le modèle des "rapports correspondants ", qui est, selon lui, plus proche de la pratique des premières communautés et de la tradition herméneutique générale. Ce modèle est signifié dans le schéma suivant (1990, p. 250):

\begin{tabular}{|c|c|c|c|c|c|c|}
\hline Jésus de Nazareth & \multirow{2}{*}{$=$} & Christ + L'Église & \multirow{2}{*}{$=$} & $\begin{array}{l}\text { Tradition } \\
\text { ecclésiale }\end{array}$ & \multirow{2}{*}{$=$} & Nous \\
\hline Son contexte & & $\begin{array}{l}\text { Contexte de } \\
\text { l'Église }\end{array}$ & & $\begin{array}{l}\text { Contexte } \\
\text { historique }\end{array}$ & & Notre contexte \\
\hline
\end{tabular}

Ou tout simplement :

\begin{tabular}{|r|l|l|}
\hline Écriture & \multirow{2}{*}{$=$} & Nous \\
${ } }$ & & Notre contexte \\
\hline
\end{tabular}

Concrètement, la médiation herméneutique se réalise à travers une relation dialectique des analyses faites avec le critère biblique, non dans le cadre d'une simple correspondance entre deux moments, mais dans un "rapport de rapports", selon le modèle des " relations correspondantes ", c'est-à-dire, la correspondance entre le rapport de l'Écriture avec le contexte du texte biblique et la relation de la théologie du politique avec le contexte actuel (BOFF, 1990, p. 252). Enfin, dans un troisième temps, l'auteur montre la logique de la foi qui mène à l'action, comprise 
comme agir politique. Si, dans les deux moments antérieurs, les " principes épistémologiques (internes) » de la théologie du politique étaient en jeu, il s'agit maintenant d'indiquer les « conditions sociales », vues de l'extérieur, soit à partir du lieu de la Praxis (1990, p. 261).

Un des plus grands mérites de $C$. Boff fut, sans aucun doute, d'expliciter et d'établir, de manière théorique, une méthode des théologies de la libération en tant que théologies du politique, en insistant sur le fait que la praxis est une partie intégrante du propre moment théologique. En d'autres mots : la praxis fait partie de la logique de la théologie elle-même. Mais là où on trouve la particularité et l'audace de cette théorie de la méthode, on en trouve aussi les limites. En vérité, celles-ci ont été mises en évidence par le changement de contexte, par l'évolution de la pensée occidentale et par la propre critique et autocritique des théologies de la libération (SCANNONE, 1994, p. 259-260 ; 268-269). Pour notre propos ici, il nous importe moins d'approfondir la teneur critique de telles limites que de montrer quelques nouveaux indicateurs qui permettent de continuer à réfléchir sur la méthode théologique.

Un premier indicateur reflète l'insuffisance de l'outil socio-analytique pour interpréter le réel dans toute sa complexité : non seulement la médiation philosophique retrouve une nouvelle valeur en théologie surtout quand il s'agit des questions éthiques et des études de la phénoménologie -, mais la mentalité et les essais interdisciplinaires révèlent aussi la contribution des sciences humaines et le besoin de la médiation d'autres formes religieuses et culturelles comme, par exemple, les rites, l'art, la littérature, etc ${ }^{20}$. Par ailleurs, dans la méthode proposée par $C$. Boff, surtout dans un premier moment de sa pensée, une certaine coupure entre la médiation socio-analytique et la médiation herméneutique (biblique) était plus accentuée, courant le risque d'une construction méthodologique en deux moments ou d'une articulation extrinsèque ${ }^{21}$. Dans une réflexion postérieure (BOFF, 1998/2012), pourtant, l'auteur non seulement reprends la question de la méthode, mais rend plus claire

20 Clodovis Boff lui-même fait attention à d'autres médiations dans sa Teoria do Método Teológico (1998).

21 “...] debemos explicitar además que esos principios y criterios ya deben operar también en el discernimiento de las mediaciones analíticas que se empleen para conocer teológicamente la realidad [...]" (SCANNONE, 1994, p. 259-260). 
sa conception tout en articulant davantage trois herméneutiques, à savoir : l'herméneutique des Écritures, du langage métaphorique et des documents du magistère (ADÃO, 2015). Car, au fond, l'herméneutique est la médiation, aussi bien de l'interprétation du contexte que des textes bibliques (RICCEUR, 1986, p. 183 et seq.). Mais, Clodovis n'hésite pas à dire que le sens ne se trouve ni au niveau du contexte ni du message : le sens spirituel est à chercher entre ces deux niveaux. Les Écritures chrétiennes proposent surtout une manière, un style, un esprit. La tâche ultime de l'herméneutique biblique est donc moins d'interpréter les Écritures par elles-mêmes que d'aider à lire la réalité présente selon les Écritures (BOFF, 1978 ; ADÃO, 2015, p. 152).

\section{Le statut herméneutique et contextuel de la foi}

L'exercice de " penser avec » Boff et Tillich nous conduit à poursuivre le chemin ouvert par l'un et par l'autre, car, comme disait ce dernier, comprendre un auteur, c'est aller au-delà de lui ${ }^{22}$. Après avoir revisité les méthodes de la corrélation et d'une tradition des théologies de la libération, nous chercherons dans l'horizon de l'herméneutique, en partant de ces deux modèles, une piste de "médiation » entre la situation et le message, entre les analyses socio-historiques et la théologie. La simple corrélation et le " rapport des rapports » exigent également un passage qui n'est jamais immédiat. La fonction d'une épistémologie théologique est, entre autres, de réfléchir après un chemin parcouru et de penser le passage d'un discours à un autre, tout en respectant l'autonomie des champs et en explicitant les logiques intrinsèques à la compréhension de la réalité et de l'expérience.

Ce mouvement correspond plus précisément au passage d'une herméneutique générale (philosophique) à une herméneutique spéciale ou régionale (théologique). Il ne s'agit cependant pas d'une simple application des règles générales de l'interprétation, étant donné que les Écritures exigent justement un « saut qualitatif » dans la manière d'interpréter le texte, grâce à « la chose du texte » (Ricœur) ou à l'événement chrétien lui-même, selon

22 "Comprendre Kant c'est aller au-delà de lui " (TILLICH, 1971, p. 273). 
lequel Jésus-Christ est à la fois le lecteur, l'interprète et l'accomplissement des Écritures (RUBENS, 2004, p. 361). Finalement, si les Écritures n'apparaissent qu'en fin d'itinéraire (de ma thèse), c'est précisément pour manifester leur caractère ultime, voire un critère décisif et déterminant. Il ne s'agit nullement de justifier la vérité des expériences en ne citant que des textes bibliques, ni de critiquer ceux-ci à partir d'une exégèse intra-bibliste. Autant l'expérience que le texte revendiquent que l'on respecte leur « altérité ». Cette altérité ne représente aucunement une menace, mais un vis-à-vis et une possibilité ouverte à un monde de relations. Le caractère chrétien de l'expérience se décide donc vis-à-vis du texte et du monde qui se déploie devant lui (RICCEUR, 1977, p. 38-39; 1969, p. 183-211).

Notre travail réalise donc un double dialogue à l'intérieur du propre paradigme herméneutique : d'une part, un dialogue avec la méthode de corrélation de Paul Tillich ; de l'autre, l'étude de la méthode des théologies latino-américaines de la libération, d'après Clodovis Boff ${ }^{23}$. Or, ces deux développements représentent des éléments significatifs pour avancer dans notre recherche d'une méthode théologique tout en révélant ses limites, soit à cause du changement de contexte, soit en raison du caractère d'" ouverture " intrinsèque à la recherche d'une méthode qui pourrait mieux rendre compte de la réalité, selon la tâche que les deux théologiens ont assumée, chacun à une époque et dans un contexte différents. Le changement de paradigmes ${ }^{24}$ en théologie suppose un double tournant, à savoir : le tournant herméneutique et celui suscité par les théologies contextuelles. Dans ce sens, Tillich est plus représentatif d'une « crise de paradigmes » de la philosophie et de la théologie : entre deux

23 Quoique la référence à ces deux noms puisse paraître arbitraire, il s'agit de mettre en évidence, d'une part, le chemin choisi dans la thèse, c'est-à-dire, “ penser avec Tillich ” une théologie du discernement de la foi et, de l'autre, le travail réalisé par Clodovis Boff dans la systématisation de la méthode des théologies latinoaméricaines de la libération. Voir aussi Taborda (1987) ; Scannone (1994).

24 Le terme "paradigme" a été utilisé parThomas S. Kuhn (1972, p. 207), dans le domaine des sciences exactes pour désigner " l'ensemble de croyances, de valeurs reconnues et de techniques communes à un groupe donné (RUBENS, 2004, p. 325-331). Voir aussi C. Palácio (1997; 2001). 
guerres, «deux mondes » et beaucoup de frontières ${ }^{25}$. Clodovis Boff ${ }^{26}$ vise à rendre compte du tournant effectué par les théologies latino-américaines aux prises avec le contexte historique. Autant le théologien allemand que le théologien brésilien ont fait valoir la situation contextuelle de leurs méthodes, bien que différemment.

Si la question du changement de paradigmes est intimement liée à l'histoire de la pensée occidentale elle-même et, plus spécifiquement, à celle qui se limite au domaine scientifique, l'acte posé par les théologies de la libération met en évidence le caractère contextuel de toute théologie. Parler d'une théologie herméneutique suppose donc un changement de paradigmes en théologie, comme l'a si bien montré Claude Geffré (1983, p. 65-90; JEANROND, 1995). Néanmoins, si l'herméneutique n'exprimait pas quelque chose d'intrinsèque à la nature même du croire, elle ne serait rien de plus qu'une simple représentation ou qu'un schéma théorique appliqué à la foi, dont l'objectif serait une mise à jour qui, quoique légitime, resterait toujours une extériorité. Mais, le tournant herméneutique devient plus radical si nous considérons, comme il se doit, que la théologie est herméneutique parce que l'acte de croire lui-même l'est aussi. L'herméneutique n'est donc pas une simple représentation, ni non plus un simple outil théorique. La foi chrétienne vit d'un constant exercice d'herméneutique, d'une exégèse des textes, d'une relecture des traditions et d'une interprétation des expériences croyantes. Dans ce sens, la raison herméneutique aide à mieux comprendre ce qui est intrinsèque à l'acte même du croire : la foi chrétienne est statutairement herméneutique (RUBENS, 2004, p. 341-343)27. Mutatis mutandis, la mise en valeur de la situation où la foi est inscrite par la manière de faire de la théologie révèle la dimension contextuelle de toute théologie en vue d'une action pastorale (GEFFRÉ, 2001, p. 31et seq.) ${ }^{28}$, de la pratique au nom de la foi et d'une

25 En effet, autant la vie que la pensée de Tillich sont situées entre de grandes " frontières ". Voir : Rubens (2004, p. 137-148) (vie) e (p. 149-231) (pensée).

26 Voir les deux ouvrages spécifiques les plus importants : Théorie et pratique: la méthode de théologies de la libération (BOFF, 1990) e Teoria do método teológico (BOFF, 1998/2012).

27 Je dois cette idée à Christoph Theobald, qui l'a développée dans son cours "Le statut herméneutique de la foi chrétienne ", donné au Centre Sèvres (Paris), de 1989 à 2000.

28 L'auteur traite de la dimension pratique de l'herméneutique au-delà d'une quête du sens, précisément à partir des questions posées par les théologies contextuelles.

Rev. Pistis Prax., Teol. Pastor., Curitiba, v. 9, n. 2, 839-860, set./dez. 2017 
prise de position politique vis-à-vis des questions de la vie sociétaire, que ce soit à l'échelle d'un village ou du monde.

Les herméneutiques biblique et théologique se définissent chacune comme une herméneutique spéciale ou régionale et suivent les règles générales de toute interprétation. Cependant ni la foi, ni le christianisme ne peuvent être à la merci de résultats issus des derniers débats herméneutiques, surtout parce que l'application des règles de l'herméneutique au corpus christianum implique un certain changement et une inversion des propres règles de l'interprétation, suscitées par la nature de la foi elle-même (RICCEUR, 1986, p. 126). Au-delà d'une corrélation entre texte et action, le changement le plus important ne se produit pas à cause de la simple application d'une méthode d'interprétation spéciale, mais en raison de la « chose même du texte » et du monde déployé devant le texte (Ricœur).

Bien qu'en fin de parcours nous soyons parvenus à quelques résultats, il faudra constater que le discernement de la foi ne se termine jamais. Car, si d'un côté, la foi chrétienne repose sur l'affirmation de la révélation pleine de Dieu en Jésus-Christ, une fois pour toutes (ephapax : $\mathrm{Hb}$ 9,12), de l'autre, un nouveau discernement doit être repris pour chaque situation nouvelle et devant quiconque nous interpelle (cf. 1Pd 3,15). Ce paradoxe, avant de se révéler à nous comme un problème, nous indique la dimension " abrahamique " de la foi et de la théologie : depuis le premier pas d'Abraham, nous sommes en marche, contemplant un ciel étoilé, mais les pieds nus (Ex 3), bien ancrés dans un contexte précis. On perçoit bien ici comment l'herméneutique de la foi implique la propre vie de ceux qui croient et pour qui la "méthode " se révèle comme chemin. Alors, il n'est pas anodin de rappeler qu'avant de recevoir le nom de chrétiens (At 11,26), ils étaient appelés disciples du chemin ou « adeptes de la voie » (At 9,2; 16,17; 18,25 et seq.).

\section{Referências}

ADÃO, F. Nossa parte da herança: os frutos de um debate teológico no Brasil. REB. Revista Eclesiástica Brasileira, v. 74, p. 264-299, 2014. 
ADÃO, F. Por uma hermenêutica bíblica pneumática: Clodovis Boff e a hermenêutica da libertação. Atualidade Teológica, Rio de Janeiro, v. 49, p. 143-156, jan./ abr. 2015.

BOFF, C. Teologia e Prática: Teologia do Político e suas mediações. Petrópolis: Vozes, 1978.

BOFF, C. Teoria do método teológico. 5. ed. Petrópolis: Vozes, 2012. (Primeira edição : 1998).

BOFF, C. Théorie et pratique: la méthode des théologies de la libération. Paris: Cerf, 1990. (Coleção Cogitatio Fidei, 157).

BRIGHENTI, A. Raíces de la epistemología y del método de la teología latinoamericana. Medellín, v. 78, p. 207-254, 1994.

ELLACURIA, I.; SOBRINO, J. Mysterium Liberationis. Conceptos fundamentales de la teología de la liberación. Madrid: Trotta, 1990. 2 v.

GAUCHET, M. Le désenchantement du monde: une histoire politique de la religion. Paris: Gallimard, 1985.

GEFFRÉ, C. Croire et interpréter. Le tournant herméneutique de la théologie. Paris: Cerf, 2001.

GEFFRÉ, C. Le christianisme au risque de l'interprétation. Paris: Cerf, 1983. (Coleção Cogitatio Fidei, 120).

GISEL, P. Pratique et Théologie. In: AMSLER, S.; et alii. Pratique et théologie: Pratiques I. Genève: Labor et Fides, 1989.

HERVIEU LEGER, D. La religion pour mémoire. Paris: éd. du Cerf, 1993.

JEANROND, W. G. Introduction à l'herméneutique théologique: Développement et signification. Paris: Cerf, 1995. (Coleção Cogitatio Fidei, 185).

KERN, W. et alii. Corso di teologia fondamentale. Brescia: Queriniana, 1990. 4. v. LAMBERIGTS, M. et al. 50 ans après le Concile Vatican II: Des théologiens du monde délibèrent. Rome: Libreria Editrice Vaticana/FIUC, 2015. 
LIBANIO, J. B. A Religião no início do milênio. São Paulo: Loyola, 2002. (Coleção Theologica, 8).

LIBANIO, J. B. Olhando para o futuro: perspectivas teológicas e pastorais do Cristianismo na América latina. São Paulo: Loyola, 2003. (Coleção Theologica, 9).

LIBANIO, J. B. Teología de la liberación: guía didáctica para su estudio. Santander: Sal Terrae, 1989.

LONERGAN, B. Method in Theology. New York: Herder and Herder, 1972.

MENDOZA-ÁLVAREZ, C. O Deus escondido da pós-modernidade. São Paulo: É Realizações Editora, 2011.

MOLTMANN, J. L’Esprit de la vie. Paris : éd. du Cerf, 1999. (Coleção Cogitatio Fidei, 212).

OLIVEIRA, P. R. de. Le catholicisme populaire en Amérique latine. Social Compass, v. 19, n. 4, p. 567-584, 1972.

PALACIO, C. Novos paradigmas ou fim de uma era teológica. In: SILVA, A. J. et al. Teologia aberta ao futuro. São Paulo: Loyola, 1997. p. 77-98.

RICCEUR, P. Le conflit des interprétations: essay d'Herméneutique. Paris: Seuil, 1969.

RICCEUR, P. Herméneutique de l'idée de Révélation: dans La Révélation. Bruxelles: Publications des Facultés universitaires Saint-Louis, 1977. p. 38-39.

RICCEUR, P. Du texte à l'action: Essais d'herméneutique II. Paris: Seuil, 1986.

RUBENS, P. Discerner la foi dans des contextes religieux ambigus. Enjeux d'une théologie du croire. Paris: Cerf, 2004. 538 p. (Coleção Cogitatio Fidei, 235).

SCANNONE, J. C. Situación de la problemática del método teológico en América latina. Medellín, v. 78, p. 255-283, jun. 1994.

SCHILLEBEECKX, E. Expérience humaine et foi en Jésus Christ. Paris: éd. du Cerf, 1981.

SCHILLEBEECKX, E. L'Histoire des hommes, récit de Dieu. Paris: éd. du Cerf, 1992. (Coleção Cogitatio Fidei, 166).

SILVA, S. La Teología fundamental entre 1970 y 1997: Panorama de panoramas y Refleciones desde América Latina. Teologia y Vida. Santiago, v. 39, p. 111-149, 1998. 
TABORDA, F. Métodos teológicos na América latina. Perspectiva Teológica, Belo Horizonte, v. 19, n. 49, p. 293-319, 1987.

THEOBALD, C. De Vatican i aux années 50. In : SESBOÜE, B. (Org.). Histoire des Dogmes: La Parole du Salut. Paris: Desclée, 1996. t. IV.

THEOBALD, C. L'Ecriture, âme de la théologie, ou le christianisme comme religion de l'interprétation. In : LAFONTAINE, R. et al. L'Ecriture, âme de la théologie. Bruxelles: éd. de l'Institut d'études théologiques, 1990. p. 111-132.

TILLICH, P. Dynamics of faith. New York: Harper and Row, 1957.

TILLICH, P. La naissance de l'esprit moderne et la théologie protestante. Paris : Éditions du Cerf, 1971.

TILLICH, P. Théologie Systematique: La vie et l'Esprit. Genève : Labor et Fides, 1991. Tome IV .

WALDENFELS, H. Manuel de théologie fondamentale. Paris: éd. du Cerf, 1990. (Coleção Cogitatio Fidei, 159). (Original: Kontextuelle Fundamentaltheologie, 1985).

Reçu : 05/03/2016

Received: 03/05/2016 Aprouvé: 26/09/2017 Approved: 09/26/2017 